\title{
Improving Walker's Algorithm to Run in Linear Time
}

\author{
Christoph Buchheim ${ }^{1}$, Michael Jünger ${ }^{1}$, and Sebastian Leipert ${ }^{2}$ \\ 1 Universität zu Köln, Institut für Informatik, \\ Pohligstraße 1, 50969 Köln, Germany \\ \{buchheim, mjuenger\}@informatik. uni-koeln.de \\ 2 caesar research center, \\ Friedensplatz 16, 53111 Bonn, Germany \\ leipert@caesar.de
}

\begin{abstract}
The algorithm of Walker [5] is widely used for drawing trees of unbounded degree, and it is widely assumed to run in linear time, as the author claims in his article. But the presented algorithm clearly needs quadratic runtime. We explain the reasons for that and present a revised algorithm that creates the same layouts in linear time.
\end{abstract}

\section{Introduction}

Since Walker presented his article [5] on drawing rooted ordered trees of unbounded degree, this topic is considered a solved problem of Automatic Graph Drawing. In 1979, Wetherell and Shannon [6] presented a linear time algorithm for drawing binary trees satisfying the following aesthetic requirements: the $\mathrm{y}$-coordinate of a node corresponds to its level, so that the hierarchical structure of the tree is displayed; the left child of a node is placed to the left of the right child, i.e., the order of the children is displayed; finally, each parent node is centered over its children. Nevertheless, this algorithm showed some deficiencies. In 1981, Reingold and Tilford [2] improved the Wetherell-Shannon algorithm by adding the following feature: each pair of isomorphic subtrees is drawn identically up to translation, i.e., the drawing does not depend on the position of a subtree within the complete tree. They also made the algorithm symmetrical: if all orders of children in a tree are reversed, the computed drawing is the reflected original one. The width of the drawing is not always minimized subject to these conditions, but it is close to the minimum in general. The algorithm of Reingold and Tilford runs in linear time, too.

Extending this algorithm to rooted ordered trees of unbounded degree in a straightforward way produces layouts where some subtrees of the tree may get clustered on a small space, even if they could be dispersed much better. This problem was solved in 1990 by the algorithm of Walker [5], which spaces out subtrees whenever possible. Unfortunately, the runtime of the algorithm presented in [5] is quadratic, in contrary to the author's assertion. In the present article, we close this gap by giving an adjustment of Walker's algorithm that does not affect the computed layouts but yields linear runtime. 
In the next section, we establish the basic notation about trees and state the aesthetic criteria guiding the algorithms to be dealt with. In Sect. 3 we explain the Reingold-Tilford algorithm. In Sect. 4, we describe the idea of Walker's algorithm and point out the non-linear parts. We improve these parts in order to get a linear time algorithm in Sect. 5 .

\section{Preliminaries}

We define a (rooted) tree as a directed acyclic graph with a single source, called the root of the tree, such that there is a unique directed path from the root to any other node. The level of a node is the length of this path. For each edge $(v, w)$, we call $v$ the parent of $w$ and $w$ a child of $v$. If $w_{1}$ and $w_{2}$ are two different children of $v$, we say that $w_{1}$ and $w_{2}$ are siblings. Each node $w$ on the path from the root to a node $v$ is called an ancestor of $v$, while $v$ is called a descendant of $w$. A leaf of the tree is a sink of the graph, i.e., a node without children. If $v_{-}$ and $v_{+}$are two nodes such that $v_{-}$is not an ancestor of $v_{+}$and vice versa, the greatest distinct ancestors of $v_{-}$and $v_{+}$are defined as the unique ancestors $w_{-}$ and $w_{+}$of $v_{-}$and $v_{+}$, respectively, such that $w_{-}$and $w_{+}$are siblings. Each node $v$ of a rooted tree $T$ induces a unique subtree of $T$ with root $v$.

In a binary tree, each node has at most two children. In an ordered tree, a certain order of the children of each node is fixed. The first (last) child according to this order is called the leftmost (rightmost) child. The left (right) sibling of a node $v$ is its predecessor (successor) in the list of children of the parent of $v$. The leftmost (rightmost) descendant of $v$ on level $l$ is the leftmost (rightmost) node on level $l$ belonging to the subtree induced by $v$. Finally, if $v_{1}$ is the left sibling of $v_{2}, w_{1}$ is the rightmost descendant of $v_{1}$ on some level $l$, and $w_{2}$ is the leftmost descendant of $v_{2}$ on the same level $l$, we call $w_{1}$ the left neighbor of $w_{2}$ and $w_{2}$ the right neighbor of $w_{1}$.

To draw a tree into the plane means to assign $\mathrm{x}$ - and $\mathrm{y}$-coordinates to its nodes and to represent each edge $(v, w)$ by a straight line connecting the points corresponding to $v$ and $w$. When drawing a rooted tree, one usually requires the following aesthetic properties:

(A1) The layout displays the hierarchical structure of the tree, i.e., the ycoordinate of a node is given by its level.

(A2) The edges do not cross each other and nodes on the same level have a minimal horizontal distance.

(A3) The drawing of a subtree does not depend on its position in the tree, i.e., isomorphic subtrees are drawn identically up to translation.

If the trees to be drawn are ordered, we additionally require the following:

(A4) The order of the children of a node is displayed in the drawing.

(A5) The algorithm works symmetrically, i.e., the drawing of the reflection of a tree is the reflected drawing of the original tree.

Here, the reflection of an ordered tree is the tree with reversed order of children for each parent node. Usually, one tries to find a layout satisfying (A1) to (A5) with a small width, i.e., with a small range of x-coordinates. 


\section{$3 \quad$ Reingold and Tilford's Algorithm}

For ordered binary trees, the first linear time algorithm satisfying (A1) to (A5) was presented by Reingold and Tilford [2]. This algorithm is easy to describe informally: it draws the tree recursively in a bottom-up sweep. Leaves are placed to an arbitrary $\mathrm{x}$-coordinate and to the $\mathrm{y}$-coordinate given by their level. After drawing the subtrees induced by the children of a parent node independently, the right one is shifted so that it is placed as close to the right of the left subtree as possible 1 Next, the parent is placed centrally above the children, this is, at the $\mathrm{x}$-coordinate given by the average $\mathrm{x}$-coordinate of the children, and at the $\mathrm{y}$-coordinate given by its level. Finally, the edges are inserted.

The Reingold-Tilford algorithm obviously satisfies (A1) to (A5). The difficult task is how to perform the steps described above in linear time. The crucial part of the algorithm is the shifting of the second subtree; solving the following problems takes quadratic runtime in total, if a straightforward algorithm is used: first, the computation of the new position of this subtree, second, the shifting of the subtree itself.

For the first problem, define the left (right) contour of a tree as the sequence of leftmost (rightmost) nodes in each level, traversed from the root to the highest level. For an illustration, see Fig. 1, where nodes belonging to the contours are shaded. To place the right subtree as close to the left one as possible, we have to compare the positions of the right contour of the left subtree with the positions of the left contour of the right subtree, for all levels occuring in both subtrees. Since each node belongs to the traversed part of the left contour of the right subtree at most for one subtree combination, the total number of such comparisons is linear for the complete tree. The runtime problem is how to traverse the contours without traversing (too many) nodes not belonging to the contours. To solve this problem, Reingold and Tilford introduce threads. For each leaf of the tree that has a successor in the same contour, the thread is a pointer to this successor. See Fig. 1 again, where the threads are represented by dotted arrows. For every node of the contour, we now have a pointer to its successor in the contour: either it is the leftmost (rightmost) child, or it is given by the thread. Finally, to keep the threads up to date, one has to add a new thread whenever two subtrees of different height are combined.

For the second problem, the straightforward algorithm would shift all nodes of the right subtree by the same value. Since this needs quadratic time in total, Reingold and Tilford attach a new value $\bmod (v)$ to each node $v$, which is called its modifier (this technique was presented by Wetherell and Shannon [6]). The position of each node is preliminary in the bottom-up traversal of the tree. When moving a subtree rooted at $v$, only $\bmod (v)$ and a preliminary x-coordinate $\operatorname{prelim}(v)$ are adjusted by the amount of shifting. The modifier of a node $v$

\footnotetext{
${ }^{1}$ For simplicity, we assume throughout this paper that all nodes have the same dimensions and that the minimal distance required between neighbors is the same for each pair of neighbors. Both restrictions can be relaxed easily, since we will always compare a single pair of neighbors
} 

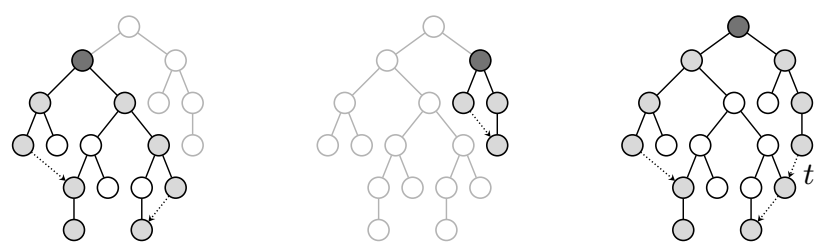

Fig. 1. Combining two subtrees and adding a new thread $t$

is interpreted as a value to be added to all preliminary $\mathrm{x}$-coordinates in the subtree rooted at $v$, except for $v$ itself. Thus, the real position of a node is its preliminary position plus the aggregated modifier modsum $(v)$ given by the sum of all modifiers on the path from the parent of $v$ to the root. To compute all real positions in linear time, the tree is traversed in a top-down fashion at the end.

When comparing contour nodes to compute the new position of the right subtree, we need the real positions of these nodes, too. For runtime reasons, we may not sum up modifiers on the paths to the root. Therefore, modifiers are used in leaves as well. A modifier of a leaf $v$ with a thread to a node $w$ stores the difference between $\operatorname{modsum}(w)$ and $\operatorname{modsum}(v)$. Since new threads are added after combining two subtrees, these modifier sums can be computed while traversing the contours of the two subtrees. We have to traverse not only the inside contours but also the outside contours for computing the modifier sums, since $v$ is a node of the outside contour. Now the aggregated modifiers can be computed as the sums of modifiers along the contours instead of the paths to the root.

\section{Walker's Algorithm}

For drawing trees of unbounded degree, the Reingold-Tilford algorithm could be adjusted easily by traversing the children from left to right, placing and shifting the corresponding subtrees one after another. However, this violates property (A5): the subtrees are placed as close to each other as possible and small subtrees between larger ones are piled to the left; see Fig. 2(a). A simple trick to avoid this effect is to add an analogous second traversal from right to left; see Fig. 2(b), and to take average positions after that. This algorithm satisfies (A1) to (A5), but smaller subtrees are usually clustered then; see Fig. 2(c).

To obtain a layout where smaller subtrees are spaced out evenly, as for example in Fig. 2(d), Walker [5] proposed the following proceeding; see Fig. 3: the subtrees of the current root are processed one after another from left to right. First, each child of the current root is placed as close to the right of its left sibling as possible. As in Reingold and Tilford's algorithm, the left contour of the current subtree is then traversed top down in order to compare the positions of its nodes to those of their left neighbors. Whenever two conflicting neighbors $v_{-}$ and $v_{+}$are detected, forcing $v_{+}$to be shifted to the right by an amount of 


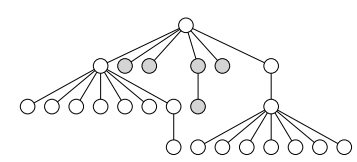

(a)

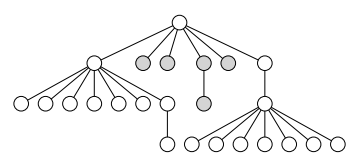

(c)

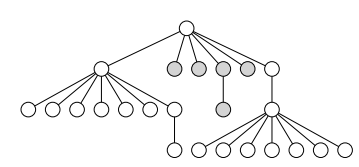

(b)

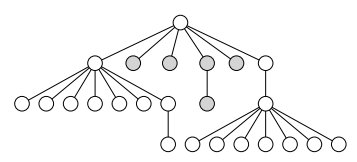

(d)

Fig. 2. Extending the Reingold-Tilford algorithm to trees of unbounded degree

shift, we apply an appropriate shift to all smaller subtrees between the subtrees containing $v_{-}$and $v_{+}$. More precisely, let $w_{-}$and $w_{+}$be the greatest distinct ancestors of $v_{-}$and $v_{+}$. Notice that both $w_{-}$and $w_{+}$are children of the current root. Let subtrees be the number of children of the current root between $w_{-}$ and $w_{+}$plus 1 . Spacing out the subtrees is shifting the subtree rooted at the $i$-th child to the right of $w_{-}$by an amount of $i \cdot$ shift/subtrees, for $i=1, \ldots$, subtrees. Observe that subtrees may be shifted several times by this algorithm, even while adding a single subtree. It is easy to see that this algorithm satisfies (A5).
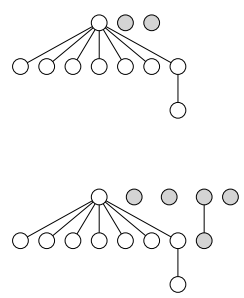
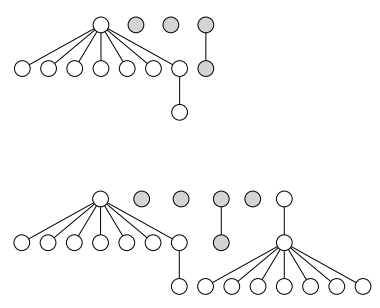

Fig. 3. Spacing out the smaller subtrees

Unfortunately, many parts of the algorithm presented in [5] do not run in linear time. Some of them are easy to improve, for example by using Reingold and Tilford's ideas, and some require new ideas. All problems concern Walker's procedure APPORTION; see pages 695-697 in [5]. In the following, we list the critical parts. In the next section, we will explain how to change these in order to obtain linear runtime.

Traversing the right contour: A recursive function GETLEFTMOST is used to find the leftmost descendant of a given node $v$ on a given level $l$. If the level 
of $v$ is $l$, the algorithm returns $v$. Otherwise, GETLEFTMOST is applied recursively to all children of $v$, from left to right. The aggregated runtime of GETLEFTMOST is not linear in general. To prove that, we present a series of trees $T_{k}$ such that the number of nodes in $T_{k}$ is $n \in \Theta\left(k^{2}\right)$, but the total number of GETLEFTMOST calls is $\Theta\left(k^{3}\right)$. Since $k \in \Theta\left(n^{1 / 2}\right)$, this shows that the total runtime of GETLEFTMOST is $\Omega\left(k^{3}\right)=\Omega\left(n^{3 / 2}\right)$. The tree $T_{k}$ is defined as follows (see Fig. 4(a) for $k=3$ ): Beginning at the root, there is a chain of $2 k$ nodes, each of the last $2 k-1$ being the right or only child of its predecessor. For $i=1, \ldots, k$, the $i$-th node in this chain has another child to the left; this child is the first node of a chain of $2(k-i)+1$ nodes. The number of nodes in $T_{k}$ is

$$
2 k+\sum_{i=1}^{k}(2(k-i)+1)=2 k+k(k-1)+k \in \Theta\left(k^{2}\right) .
$$

Now, for each $i=0, \ldots, k-1$, we have to combine two subtrees when visiting the node on the right contour of $T_{k}$ on level $i$. In this combination, the highest common level of the subtrees is $2 k-i-1$, and by construction of $T_{k}$ we always have to apply GETLEFTMOST to every node of the right subtree up to this level. The number of these nodes is

$$
k-i+\sum_{j=0}^{k-i-1}(2 j)=(k-i)+(k-i)(k-i-1)=(k-i)^{2},
$$

hence the total number of GETLEFTMOST calls for all combinations is

$$
\sum_{i=0}^{k-1}(k-i)^{2}=\sum_{i=1}^{k} i^{2}=k(k+1)(2 k+1) / 6 \in \Theta\left(k^{3}\right) .
$$

Finding the ancestors and summing up modifiers: This part of the algorithm is obviously quadratic. When adjusting the current subtree to the left subforest, the greatest distinct ancestors of the possibly conflicting neighbors are computed for each level by traversing the graph up to the current root, at the same time computing the modifier sums. Since the distance of the levels grows linearly, the total number of steps is in $\Omega\left(n^{2}\right)$.

Counting and shifting the smaller subtrees: When shifting the current subtree to the right because of a conflict with a subtree to the left, the procedure APPORTION also shifts all smaller subtrees in-between immediately. Furthermore, the number of these subtrees is computed by counting them one by one. Both actions have an aggregated runtime of $\Omega\left(n^{3 / 2}\right)$, as the following example shows. Let the tree $T^{k}$ be constructed as follows (see Fig. 4(b) for $k=3$ ): add $k$ children to the root. The $i$-th child, counted $i=1, \ldots, k$ from left to right, is root of a chain of $i$ nodes. Between each pair of these children, add $k$ children as leaves. The leftmost child of the root has $2 k+5$ children, and up to level $k-1$, every rightmost child of the $2 k+5$ children has again $2 k+5$ children. The number 
of nodes of $T^{k}$ is

$$
1+\sum_{i=1}^{k} i+(k-1) k+(k-1)(2 k+5) \in \Theta\left(k^{2}\right) .
$$

Furthermore, by construction of the left subtree, adding the $i$-th subtree chain for $i=2, \ldots, k$ results in a conflict with the left subtree on level $i$. Hence all $(i-1)(k+1)-1$ smaller subtrees between the two conflicting ones are counted and shifted. Thus, the total number of counting and shifting steps is

$$
\sum_{i=2}^{k}((i-1)(k+1)-1)=(k+1) k(k-1) / 2-k+1 \in \Theta\left(k^{3}\right) .
$$

As in the last example, we derive that counting and shifting needs $\Omega\left(n^{3 / 2}\right)$ time in total.

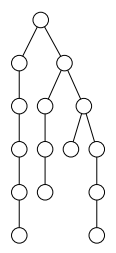

(a) $T_{3}$

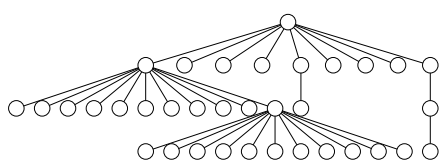

(b) $T^{3}$

Fig. 4. Examples proving the non-linear runtime of Walker's algorithm

\section{Improving Walker's Algorithm}

In this section, we explain how to improve the algorithm of Walker to run in linear time without affecting the computed layouts. For a closer look, see [1], where we present the complete revised algorithm in a pseudocode style as well as an experimental runtime comparison of both versions.

Traversing the contours and summing up modifiers: This can be done exactly as in the case of binary trees by using threads; see Sect. 3. The fact that the left subforest is no tree in general does not create any additional difficulty.

Finding the ancestors: The problem of finding the greatest distinct ancestors $w_{-}$ and $w_{+}$of two nodes $v_{-}$and $v_{+}$can be solved by the algorithm of Schieber and Vishkin [3]. For each pair of nodes, this algorithm can determine the greatest distinct ancestors in constant time, after an $O(n)$ preprocessing step. However, 
in our application, a much simpler algorithm can be applied. First observe that we know the right ancestor $w_{+}$anyway; it is just the root of the current subtree. Furthermore, as $v_{+}$is always the right neighbor of $v_{-}$in our algorithm, the left one of the greatest distinct ancestors only depends on $v_{-}$. Thus we may shortly call it the ancestor of $v_{-}$in the following. We use a node pointer ancestor $(x)$ for each node $x$ to save its ancestor and initialize it to $x$ itself. Observe that this value is not correct for rightmost children, but we do not need the correct value $w_{-}$of ancestor $\left(v_{-}\right)$until the right neighbor $v_{+}$of $v_{-}$is added, i.e., until the current root is the parent node of $w_{-}$. Hence assume that we are placing the subtrees rooted at the children of $v$ from left to right. Since tracing all ancestor $(x)$ consumes too much time, we use another node pointer defaultAncestor. Our aim is to have the following property $(*)$ for all nodes $v_{-}$on the right contour of the left subforest after each subtree addition: if ancestor $\left(v_{-}\right)$is up to date, i.e., is a child of $v$, then it points to the correct ancestor $w_{-}$of $v_{-}$; otherwise, the correct ancestor is defaultAncestor. We start with placing the first subtree, rooted at $w$, which does not require any ancestor calculations. After that, we set defaultAncestor to $w$. Since all pointers ancestor $(x)$ of the left subtree either point to $w$ or to a node of a higher level, the desired property $(*)$ holds, see Fig. 5(a). After placing the subtree rooted at another child $w^{\prime}$ of $v$, we distinguish two cases: if the subtree rooted at $w^{\prime}$ is smaller than the left subforest, we can actualize ancestor $(x)$ for all nodes $x$ on its right contour by setting it to $w^{\prime}$. By this, we obviously keep $\left(^{*}\right)$; see Fig. 5(b). Otherwise, if the new subtree is larger than the left subforest, we may not do the same because of runtime. But now it suffices to set defaultAncestor to $w^{\prime}$, since again all pointers ancestor $(x)$ of the subtree induced by $w^{\prime}$ either point to $w^{\prime}$ or to a node of a higher level, and all other subtrees in the left subforest are hidden. Hence we have $\left(^{*}\right)$ again; see Fig. 5(c).

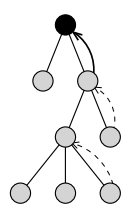

(a)

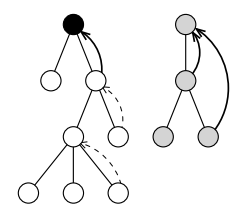

(b)
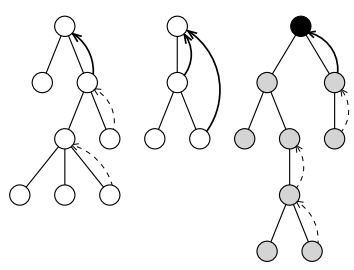

(c)

Fig. 5. Adjusting ancestor pointers when adding new subtrees: the pointer ancestor $(x)$ is represented by a solid arrow if it is up to date and by a dashed arrow if it is expired. In the latter case, the defaultAncestor is used and drawn black. When adding a small subtree, all ancestor pointers ancestor $(x)$ of its right contour are updated. When adding a large subtree, only defaultAncestor is updated 
Counting the smaller subtrees: For that, we just have to number the children of each node consecutively; then the number of smaller subtrees between the two greatest distinct ancestors $w_{-}$and $w_{+}$is the number of $w_{+}$minus the number of $w_{-}$minus 1 . Hence it can be computed in constant time (after a linear time preprocessing step to compute all child numbers).
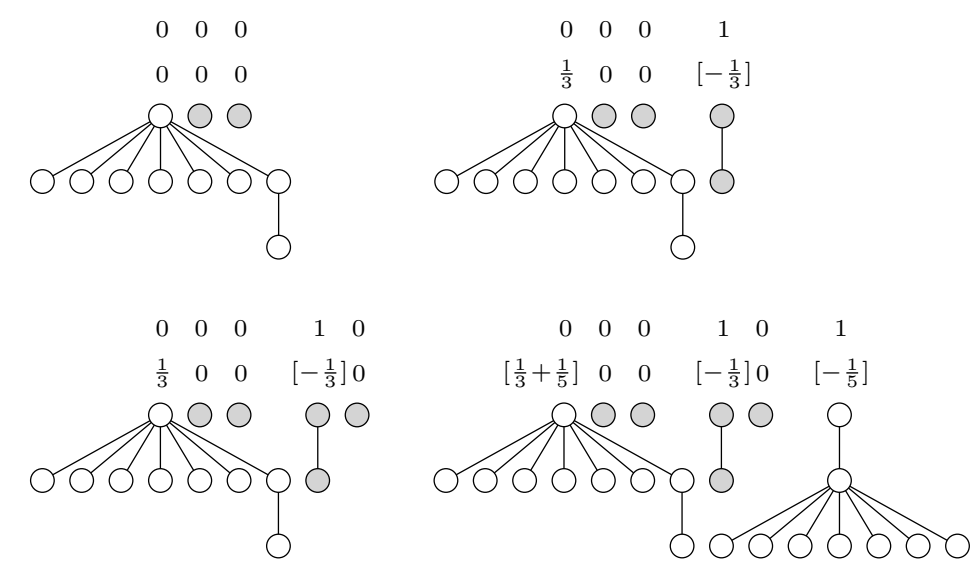

Fig. 6. Aggregating the shifts: the top number at node $x$ indicates the value of $\operatorname{shift}(x)$, and the bottom number indicates the value of change $(x)$

Shifting the smaller subtrees: In order to get a linear runtime, we will shift each subtree at most once when it is not the currently added subtree. The currently added subtree, however, may be shifted whenever it conflicts with a subtree to the left, using the fact that shifting a single subtree is done in constant time (recall that we only have to adjust $\operatorname{prelim}\left(w_{+}\right)$and $\bmod \left(w_{+}\right)$). Furthermore, shifting the current subtree immediately is necessary to keep the right contour of the left subforest up to date. All shiftings of non-current subtrees are performed in a single traversal after all subtrees of the current root have been placed. To memorize the shiftings at the moment they arise, we use real numbers $\operatorname{shift}(x)$ and change $(x)$ for each node $x$ and set both to zero at the beginning. Assume that the subtree rooted at $w_{+}$is the subtree currently placed, and that a conflict with the subtree rooted at $w_{-}$forces the current subtree to move to the right by an amount of shift. Let subtrees be the number of subtrees between $w_{-}$and $w_{+}$, plus 1 . According to Walker's idea, the $i$-th of these subtrees has to be moved by $i \cdot$ shift/subtrees. We save this by increasing shift $\left(w_{+}\right)$by shift, decreasing change $\left(w_{+}\right)$by shift/subtrees, and increasing change $\left(w_{-}\right)$by shift/subtrees. The interpretation of this is the following: to the left of node $w_{+}$, the nodes are shifted by an amount initialized to shift, but this amount starts decreasing by shift/subtrees per subtree at node $w_{+}$and ends decreasing at $w_{-}$, where it is zero. The trick is to aggregate the shifts: since the decrease in the amount of 
shifting is linear, we can add all these decreases in one array; see Fig. 6 for an example. Finally, we execute all shifts in a single traversal of the children of the current root as follows, see Fig. 7: we use two real values shift and change to store the shifts and the decreases of shift per subtree, respecti国ly, and set both to zero at the beginning. Then we traverse the children from right to left. When visiting child $v$, we move $v$ to the right by shift (i.e., we increase $\operatorname{prelim}(v)$ and $\bmod (v)$ by shift), increase change by change $(v)$, and increase shift by $\operatorname{shift}(v)$ and by change. Then we go on to the left sibling of $v$. It is easy to see that this algorithm shifts each subtree by the correct amount.

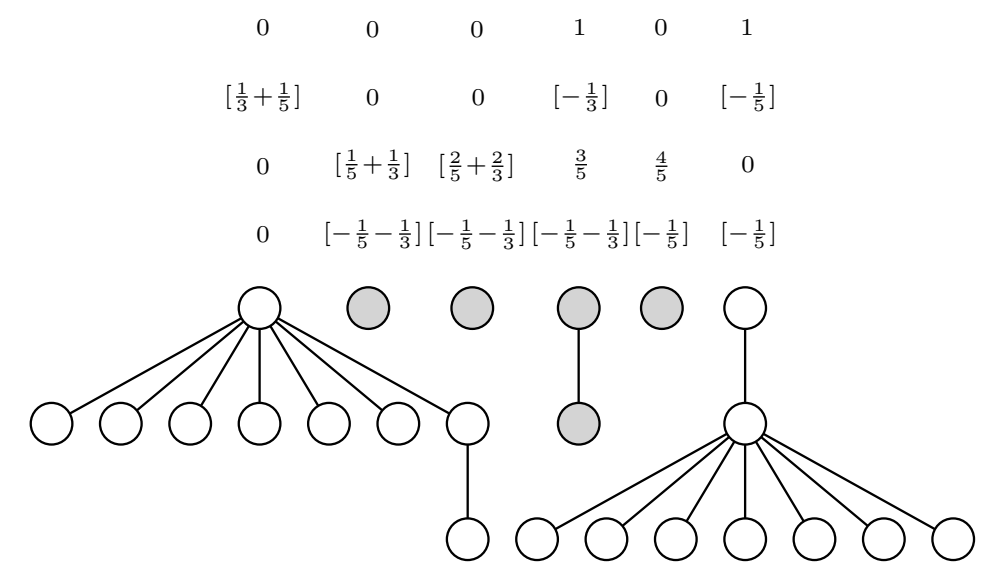

Fig. 7. Executing the shifts: the new numbers at node $x$ indicate the values of shift and change before shifting $x$, respectively

\section{References}

1. C. Buchheim, M. Jünger, and S. Leipert. Improving Walker's algorithm to run in linear time. Technical Report zaik2002-431, ZAIK, Universität zu Köln, 2002.

2. E. Reingold and J. Tilford. Tidier drawings of trees. IEEE Transactions on Software Engineering, 7(2):223-228, 1981.

3. B. Schieber and U. Vishkin. On finding lowest common ancestors: Simplification and parallelization. In Proceedings of the Third Aegean Workshop on Computing, volume 319 of Lecture Notes in Computer Science, pages 111-123, 1988.

4. K. Supowit and E. Reingold. The complexity of drawing trees nicely. Acta Informatica, 18(4):377-392, 1983.

5. J. Walker II. A node-positioning algorithm for general trees. Software - Practice and Experience, 20(7):685-705, 1990.

6. C. Wetherell and A. Shannon. Tidy drawings of trees. IEEE Transactions on Software Engineering, 5(5):514-520, 1979. 